\title{
Improvement of immune functions in elderly men and women after 3 months of vitamin $C$ and vitamin $C$ plus $E$ supplementation
}

\author{
M. De La Fuente ${ }^{1}$, O. Hernández ${ }^{1}$, C. Sanchez ${ }^{1}$, F. Arnalich ${ }^{2}$ and A. Hernanz ${ }^{3}$ \\ ${ }^{1}$ Department of Physiology. Faculty of Biology, Complutense University of Madrid, ${ }^{2}$ Internal Medicine Department and \\ ${ }^{3}$ Biochemistry Department, Hospital Universitario La Paz, Madrid, Spain
}

\begin{abstract}
With ageing, a chronic oxidative process, the immune response is impaired. This immunosenescence, in which an alteration of the redox state of the immune cells appears (with an increase in oxidant levels and a decrease in antioxidant defences), is involved with the rate of ageing $^{(1)}$. Leucocyte functions are good health markers and longevity predictors ${ }^{(1)}$ and nutrition has been proposed as one strategy of life style, which help to maintain an excellent immune function with aging ${ }^{(2)}$. The aim of this work was to study the effects of daily oral administration of the antioxidant vitamin C $(500 \mathrm{mg})$, or both vitamin C $(500 \mathrm{mg})$ and vitamin E $(200 \mathrm{mg})$ on several blood neutrophil functions (adherence, chemotaxis, phagocytosis, and superoxide anion levels) and lymphocyte functions (adherence, chemotaxis, proliferation, interleukin-2 secretion and natural killer activity) in healthy elderly men and women. These parameters were analysed before supplementation, after 3 months of supplementation, and 6 months after the end of supplementation (post-supplementation). In addition, adult subjects were used as the control age group. The results showed that vitamin $\mathrm{C}$ improved these immune parameters in elderly subjects, bringing their values close to those of adults. These effects were maintained after 6 months without supplementation in several functions. Similar effects were found in elderly supplemented with both vitamin C and E. Thus, a short period of vitamin C or vitamin C and $\mathrm{E}$ administration, at the doses used, improving the immune functions, could contribute to increase healthy longevity in elderly individuals.
\end{abstract}

This work was supported by MICINN (BFU2011-30336), UCM (910379ENEROINN) grants, RETICEF (RD06/0013/0003) from ISCIII.

1. De la Fuente M \& Miquel J (2009) Current Pharm Des 15 (26), 3003-3026.

2. De la Fuente M, Cruces J, Hernandez O \& Ortega E (2011) Current Pharm. Des 17 (36), 3966-3993. 